\title{
A Study of NK Landscapes' Basins and Local Optima Networks
}

\author{
Gabriela Ochoa \\ Automated Scheduling, \\ Optimisation and Planning \\ School of Computer Science \\ University of Nottingham, UK \\ gxo@cs.nott.ac.uk
}

\author{
Marco Tomassini \\ Information Systems \\ Department \\ University of Lausanne \\ Lausanne, Switzerland \\ Marco.Tomassini@unil.ch \\ Christian Darabos \\ Information Systems \\ Department \\ University of Lausanne \\ Lausanne, Switzerland \\ Christian.Darabos@unil.ch
}

\author{
Sebástien Vérel \\ Laboratoire I3S \\ CNRS-University of Nice \\ Sophia Antipolis, France \\ verel@i3s.unice.fr
}

\begin{abstract}
We propose a network characterization of combinatorial fitness landscapes by adapting the notion of inherent networks proposed for energy surfaces [ []. We use the well-known family of $N K$ landscapes as an example. In our case the inherent network is the graph where the vertices are all the local maxima and edges mean basin adjacency between two maxima. We exhaustively extract such networks on representative small $N K$ landscape instances, and show that they are 'small-worlds'. However, the maxima graphs are not random, since their clustering coefficients are much larger than those of corresponding random graphs. Furthermore, the degree distributions are close to exponential instead of Poissonian. We also describe the nature of the basins of attraction and their relationship with the local maxima network.
\end{abstract}

\section{Categories and Subject Descriptors}

I.2.8 [Artificial Intelligence]: Problem Solving, Control Methods, and Search-Heuristic methods; G.2.2 [Discrete Mathematics]: Graph Theory-Network problems

\section{General Terms}

Algorithms, Measurement, Performance

\section{Keywords}

Landscape Analysis, Network Analysis, Complex Networks, Local Optima, $N K$ Landscapes

Permission to make digital or hard copies of all or part of this work for personal or classroom use is granted without fee provided that copies are not made or distributed for profit or commercial advantage and that copies bear this notice and the full citation on the first page. To copy otherwise, to republish, to post on servers or to redistribute to lists, requires prior specific permission and/or a fee.

GECCO'08, July 12-16, 2008, Atlanta, Georgia, USA.

Copyright 2008 ACM 978-1-60558-131-6/08/07 ...\$5.00.

\section{INTRODUCTION}

A fitness landscape of a combinatorial problem can be seen as a graph whose vertices are the possible configurations. If two configurations can be transformed into each other by a suitable operator move, then we can trace an edge between them. The resulting graph, with an indication of the fitness at each vertex, is a representation of the given problem fitness landscape. Doye [\$, 6] has recently introduced a useful simplification of the fitness landscape graph for the energy landscapes of atomic clusters. The idea consists in taking as vertices of the graph not all the possible configurations, but only those that correspond to energy minima. For atomic clusters these are well-known, at least for relatively small assemblages. Two minima are considered connected, and thus an edge is traced between them, if the energy barrier separating them is sufficiently low. In this case there is a transition state, meaning that the system can jump from one minimum to the other by thermal fluctuations going through a saddle point in the energy hypersurface. The values of these activation energies are mostly known experimentally or can be determined by simulation. In this way, a network can be built which is called the "inherent structure" or "inherent network" in [5]. We use a modification of this idea for studying the well-known $N K$ combinatorial landscapes. In our case, a vertex of the graph is a local maximum, and there is an edge between two maxima if they lay on adjacent basins.

In the context of meta-heuristics, it is important to identify the features of landscapes that would influence the effectiveness of heuristic search. Such knowledge may be helpful for both predicting the performance and improving the design of meta-heuristics. Among the features of landscapes known to have a strong influence on heuristic search, is the number and distribution of local optima in the search space. An interesting property of combinatorial landscapes, which has been observed in many different studies, is that on average, local optima are very much closer to the global optimum than are randomly chosen points, and closer to each other than random points would be. In other words, the local optima are not randomly distributed, rather they tend to be clustered in a "central massif" (or "big valley" if we are minimising). This globally convex landscape structure has been observed in the $N K$ family of landscapes [11], and in many combinatorial optimisation problems, 
such as the traveling salesman problem [2], graph bipartitioning [13], and flowshop scheduling [16].

In this study we seek to provide fundamental new insights into the structural organization of the local optima in combinatorial landscapes, particularly into the connectivity and characteristics of their basins of attraction, using $N K$ landscapes as a case study. To achieve this, we first map the landscape onto a network, and then analyze the topology of this network for a number of small $N K$ landscape instances for which complete networks can be obtained. Our analysis is inspired, in particular, by the work of Doye [ [ 1 , 6] on energy landscapes, and in general, by the field of complex networks [14, 20, 21]. The study of complex networks has already permeated the evolutionary computation field. Specifically, in the study of scientific collaborations [3, 12], the structure of a population in cellular evolutionary algorithms [9, 10, 15, and the evolution of networks of cellular automata [19]. However, our study is the first attempt, to our knowledge, of using network analysis techniques in connection with the study of fitness landscapes and problem difficulty in combinatorial optimization.

The next section introduces the study of complex networks, and describes the main features of small-world and scale-free networks. Section 3 describes how landscapes are mapped onto networks, and includes the relevant definitions and algorithms. The empirical network analysis of our selected $N K$ landscape instances is presented in Section 4, whilst Section 5 gives our conclusions and ideas for future work.

\section{COMPLEX NETWORKS}

The recent interest in the study of networks and networked systems was influenced by the seminal paper by Watts and Strogatz [21], who showed that many real-world networks are neither completely ordered nor completely random, but rather exhibit important properties of both. Some of these network properties can be quantified by simple statistics such as the clustering coefficient $C$, which is a measure of local density, and the average shortest path length $l$, which is a global measure of separation. It has been shown in recent years that many social, biological, and man-made system show what has been called a small-world topology [21], in which nodes are highly clustered yet the path length between them is small.

A second important aspect in the study of networks has been the realization that in many real-world networks, the distribution of the number of neighbours (the degree distribution) is typically right-skewed with a "heavy tail", meaning that most of the nodes have less-than-average degree whilst a small fractions of hubs have a large number of connections. These qualitative description can be described mathematically by a power-law [1], which has the asymptotic form $p(k) \sim k^{-\alpha}$. This means that the probability of a randomly chosen point having a degree $k$ decays like a power of $k$, where the exponent $\alpha$ (typically in the range $[2,3]$ ) determines the rate of decay. A distinguishing feature of power-law distributions is that when plotted on a double logarithmic scale, a powerlaw appears as a straight line with negative slope $\alpha$. This behavior contrasts with a normal distribution which would curve sharply on a log-log plot, such that the probability of a node having a degree greater than a certain "cutoff" value is nearly zero. The mean would then trivially represent a characteristic scale for the network degree distribution. Since networks with power-low degree distribution lack any such cutoff value, at least in theory, they are often called scale-free networks [2]]. Examples of such scale-free networks are the world-wide-web, the internet, scientific collaboration and citation networks, and biochemical networks.

\section{LANDSCAPES AS NETWORKS}

To model a physical energy landscape as a network, Doye [6] needed to decide first on a definition both of a state of the system and how two states were connected. The states and their connections will then provide the nodes and edges of the network. For systems with continuous degrees of freedom, the author achieved this through the 'inherent structure' mapping [18]. In this mapping each point in configuration space is associated with the minimum (or 'inherent structure') reached by following a steepest-descent path from that point. This mapping divides configuration into basins of attraction surrounding each minimum on the energy landscape.

We use a modification of this idea for the $N K$ family of binary landscapes, which indeed can be applied to any combinatorial landscape. In our case, the vertexes of the graph are the local maxima of the landscape, obtained exhaustively by running a bestimprovement local search algorithm (see Algorithm 1) from every configuration of the search space. The edges in the network connect local optima of adjacent basins of attraction. An illustration for a model 2D landscape can be seen in Figure 1, which is inspired by a similar figure appearing in 5,6 . Here, we illustrate a network of local maxima (instead of local minima). A more formal definition of our inherent networks is given in Section 3.1. As it was the case in the study on physical energy landscapes [ multiple edges, or weights in the edges. This may be a factor to consider in future work.

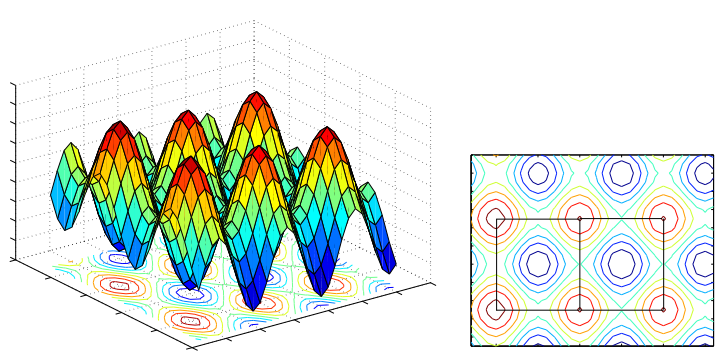

Figure 1: A model of a 2D landscape (left), and a contour plot of the local optima partition of the configuration space into basins of attraction surrounding maxima and minima (right). A simple regular network of six local maxima can be observed.

Note that while a physical energy landscape is formally a continuous landscape, ours are strictly combinatorial, i.e. discrete and finite. Moreover, the energy landscape of a stable atomic cluster, crystal or molecule is relatively smooth and easy to search and has been called a "funnel" landscape [5]. In contrast, in $N K$ landscapes one can continuously vary the intrinsic landscape difficulty by changing the value of $K$. As a result, we shall see that $N K$ landscapes show a number of different behaviors depending on $K$ for a given $N$, and these different behaviors are reflected on their inherent networks. Indeed, $N K$ landscapes can be seen as analogous to those of spin-glasses [11, 17]. In contrast to atomic cluster energy landscapes, spin glass landscapes may show frustration, i.e. configurations that must respect conflicting constraints, and solving for the ground state of the system that is, the minimum energy configuration is an NP-hard problem. Similar consequences are caused by the introduction of epistatic interactions through the increase of the $K$ value in $N K$ landscapes.

Below we present the relevant formal definitions and algorithms to obtain our combinatorial analogous of an energy landscape inherent network. 


\subsection{Definitions and Algorithms}

Definition : Fitness landscape

A landscape is a triplet $(S, V, f)$ where $S$ is a set of potential solutions i.e. a search space, $V: S \longrightarrow 2^{S}$, a neighborhood structure, is a function that assigns to every $s \in S$ a set of neighbours $V(s)$, and $f: S \longrightarrow R$ is a fitness function that can be pictured as the height of the corresponding potential solutions.

In our study, the search space is composed by binary strings of length $N$, therefore its size is $2^{N}$. The neighborhood is defined by the minimum possible move on a binary search space, that is, the 1-move or bit-flip operation. In consequence, for any given string $s$ of length $N$, the neighborhood size is $|V(s)|=N$. Notice that in $N K$ landscapes, two neighboring solutions never have the same fitness value. Therefore, neutrality is not present. Landscapes with neutrality will be considered in future work.

Definition: Local Optimum.

A local optimum is a solution $s^{*}$ such that $\forall s \in V\left(s^{*}\right), f(s)<$ $f\left(s^{*}\right)$.

The LocalSearch algorithm to determine the local optima and therefore define the basins of attraction, is given below:

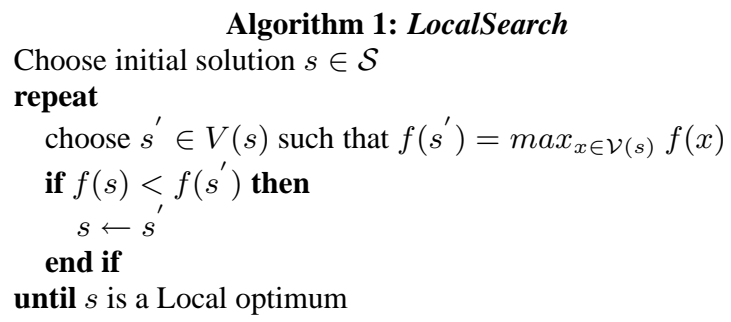

The LocalSearch algorithm defines a mapping from the search space $S$ to the set of locally optimal solutions $S^{*}$. We therefore define a basin of attraction as follows:

Definition : Basin of attraction.

The basin of attraction of a local optimum $i$ is the set $b_{i}=\{s \in$ $S \mid \operatorname{LocalSearch}(s)=i\}$. The size of the basin of attraction of a local optima $i$ is the cardinality of $b_{i}$.

We then define the inherent network, or network of local optima as:

Definition : Local optima network.

The local optima network $G=\left(S^{*}, E\right)$ is the graph where the nodes are the local optima, and there is an edge $e_{i j} \in E$ between two local optima $i$ and $j$ if there is at least a pair of direct neighbors (1-bit apart) $s_{i}$ and $s_{j}$, such that $s_{i} \in b_{i}$ and $s_{j} \in b_{j}$. That is, if there exists a pair of direct neighbors solutions $s_{i}$ and $s_{j}$, one in each basin $\left(b_{i}\right.$ and $\left.b_{j}\right)$

\section{EMPIRICAL NETWORK ANALYSIS}

\subsection{Experimental Setting}

The $N K$ family of landscapes [11] is a problem-independent model for constructing multimodal landscapes that can gradually be tuned from smooth to rugged. In the model, $N$ refers to the number of (binary) genes in the genotype (i.e. the string length) and $K$ to the number of genes that influence a particular gene. By increasing the value of $K$ from 0 to $N-1, N K$ landscapes can be tuned from smooth to rugged. The $k$ variables that form the context of the fitness contribution of gene $s_{i}$ can be chosen according to different models. The two most widely studied models are the random neighborhood model, where the $k$ variables are chosen randomly according to a uniform distribution among the $n-1$ variables other than $s_{i}$, and the adjacent neighborhood model, in which the $k$ variables that are closest to $s_{i}$ in a total ordering $s_{1}, s_{2}, \ldots, s_{n}$ (using periodic boundaries). No significant differences between the two models were found in [11] in terms of global properties of the respective families of landscapes, such as mean number of local optima or autocorrelation length. Therefore, we explore here the adjacent neighborhood model, leaving the random model for future analysis.

In order to avoid sampling problems that could bias the results, we used the largest values of $N$ that can still be analyzed exhaustively with reasonable computational resources. We thus extracted the local optima networks of landscape instances with $N=16,18$, and $K=2,4,6, \ldots, N-2, N-1$. For each pair of $N$ and $K$ values, 30 instances were explored. Therefore, the networks statistics reported below represent the average behaviour of 30 independent instances.

\subsection{General Network Statistics}

Table 1 reports the average of the network properties measured on $N K$ landscapes for $N=16,18$ and all even $K$ values; $K=$ $N-1$ is also given. Values are averages over 30 randomly generated landscapes. $\bar{n}_{v}$ and $\bar{n}_{e}$ are, respectively, the mean number of vertices and the mean number of edges of the graph for a given $K$ rounded to the next integer. $\bar{C}$ is the average of the mean clustering coefficients ${ }^{1}$ over all the generated landscapes. $C_{r}$ is the average clustering coefficient of a random graph with the same number of vertices and mean degree. $\bar{z}$ is the average of the mean degrees. $\bar{l}$ is the average of the mean path lengths over all landscape instances. The last column contains the average degree assortativity coefficient $\bar{a}$, which measures whether nodes with similar degrees tend to pair up with each other. The assortativity coefficient is computed according to [14].

Notice that the mean number of vertexes $\left(\bar{n}_{v}\right)$ confirms that the number of local optima (and thus the search difficulty) increases with the value of $K$. Some other interesting inferences can be drawn from these metrics. First of all, looking at the $\bar{l}$ values one can conclude that the maxima networks are small worlds for all values of $K$ since the growth of $\bar{l}$ is bounded by a function $O\left(\log n_{v}\right)$. In a sense, this is not surprising as the whole configuration space spans the binary hypercube $\{0,1\}^{N}$ of degree $N$ with $2^{N}$ vertices, which has maximum distance (diameter) $d=\log 2^{N}$, i.e. 16 and 18 for our studied instances. However, while the base configuration space has constant degree for any node, the maxima network are degree-inhomogeneous (see next section) and have clustering coefficients well above those of equivalent random graphs, showing that there is local structure in the networks. For both $N=16$ and 18 , the mean degree $\bar{z}$ first increases with $K$ and then goes down again for $K>8$. The assortativity coefficients are always very small which means that there is almost no correlation between the degrees of neighboring nodes. For easy energy landscapes, Doye found that the networks were slightly disassortative [6].

\subsection{Degree Distributions}

The degree distribution function $p(k)$ of a graph represents the probability that a randomly chosen node has degree $k$ [14]. Random graphs are characterized by a $p(k)$ of Poissonian form, while

\footnotetext{
${ }^{1}$ The clustering coefficient $C_{i}$ of a node $i$ is defined as $C_{i}=$ $2 E_{i} / k_{i}\left(k_{i}-1\right)$, where $E_{i}$ is the number of edges in the neighborhood of $i$. Thus $C_{i}$ measures the amount of "cliquishness" of the neighborhood of node $i$ and it characterizes the extent to which nodes adjacent to node $i$ are connected to each other. The clustering coefficient of the graph is simply the average over all nodes: $C=\frac{1}{N} \sum_{i=1}^{N} C_{i}[14$.
} 
Table 1: Network properties of $N K$ landscapes for $N=16,18$ and all even $K$ values; $K=N-1$ is also given. Values are averages over 30 randomly generated landscapes, standard deviations are shown as subscripts. $n_{v}$ and $n_{e}$ represent the number of vertexes and edges (rounded to the next integer), $\bar{C}$, the mean clustering coefficient, whilst $C_{r}$ is the clustering coefficient of a random graph with the same number of vertexes and mean degree, which is $C_{r} \simeq \bar{z} / \bar{n}_{v} . \bar{z}$ represent the mean degree, $\bar{l}$ the mean path length , and $\bar{a}$ the degree assortativity coefficient.

\begin{tabular}{|c|c|c|c|c|c|c|c|}
\hline \multicolumn{8}{|c|}{$N=16$} \\
\hline$K$ & $\bar{n}_{v}$ & $\bar{n}_{e}$ & $C$ & $C_{r}$ & $\overline{\bar{z}}$ & $l$ & $\bar{a}$ \\
\hline 2 & $33_{15}$ & $261_{166}$ & $0.68_{0.095}$ & $0.507_{0.1536}$ & 14.553 .826 & $1.54_{0.182}$ & $-0.0007_{0.00591}$ \\
\hline 4 & 17833 & $6,334_{1646}$ & 0.660 .036 & 0.4060 .0615 & 70.486 .615 & $1.60_{0.062}$ & $-0.0162_{0.00467}$ \\
\hline 6 & $460_{29}$ & $26,414_{2035}$ & $0.55_{0.013}$ & $0.250_{0.0150}$ & $114.76_{3.033}$ & $1.75_{0.016}$ & $-0.0237_{0.00283}$ \\
\hline 8 & 89033 & 56,0221951 & $0.44_{0.008}$ & $0.139_{0.0061}$ & 124.521 .800 & 1.880 .008 & $-0.0219_{0.00250}$ \\
\hline 10 & $1,470_{34}$ & $86,446_{1766}$ & $0.36_{0.006}$ & $0.080_{0.0023}$ & $117.62_{1.137}$ & $2.00_{0.009}$ & $-0.0170_{0.00182}$ \\
\hline 12 & $2,254_{32}$ & $117,085_{1111}$ & $0.30_{0.003}$ & $0.046_{0.0009}$ & $103.91_{0.695}$ & $2.19_{0.012}$ & $-0.0122_{0.00104}$ \\
\hline 14 & $3,264_{29}$ & $146,390_{1025}$ & $0.26_{0.002}$ & $0.027_{0.0003}$ & $89.70_{0.349}$ & $2.47_{0.009}$ & $-0.0092_{0.00064}$ \\
\hline 15 & $3,868_{33}$ & $160,690_{829}$ & $0.25_{0.002}$ & $0.021_{0.0003}$ & $83.09_{0.469}$ & $2.58_{0.007}$ & $-0.0086_{0.00059}$ \\
\hline \multicolumn{8}{|c|}{$\bar{N} N=18$} \\
\hline 2 & $50_{25}$ & 478342 & $0.62_{0.106}$ & $0.414_{0.1697}$ & 17.084 .930 & 1.660 .210 & $0.00390_{0.00530}$ \\
\hline 4 & $330_{72}$ & $17,576_{4898}$ & $0.61_{0.044}$ & $0.332_{0.0573}$ & $105.39_{8.106}$ & $1.67_{0.058}$ & $-0.0168_{0.00495}$ \\
\hline 6 & $994_{73}$ & 93,0438588 & $0.51_{0.016}$ & $0.189_{0.0115}$ & $187.07_{4.650}$ & $1.82_{0.012}$ & $-0.0279_{0.00321}$ \\
\hline 8 & 2,09370 & $214,844_{6793}$ & $0.41_{0.007}$ & $0.098_{0.0038}$ & $205.29_{2.615}$ & $1.92_{0.006}$ & $-0.0263_{0.00184}$ \\
\hline 10 & $3,619_{61}$ & $348,761_{5275}$ & $0.33_{0.004}$ & $0.053_{0.0011}$ & $192.76_{1.150}$ & $2.05_{0.009}$ & $-0.0199_{0.00127}$ \\
\hline 12 & $5,657_{59}$ & 476,6143416 & $0.27_{0.002}$ & $0.030_{0.0005}$ & $168.50_{1.003}$ & $2.29_{0.012}$ & $-0.0141_{0.00072}$ \\
\hline 14 & 8,35260 & $594,902_{2459}$ & $0.23_{0.001}$ & $0.017_{0.0002}$ & $142.46_{0.652}$ & $2.56_{0.007}$ & $-0.0102_{0.00044}$ \\
\hline 16 & $11,797_{63}$ & $707,326_{2296}$ & $0.21_{0.001}$ & $0.010_{0.0001}$ & $119.92_{0.368}$ & $2.72_{0.003}$ & $-0.0080_{0.00036}$ \\
\hline 17 & 13,79577 & 762,1972299 & $0.20_{0.001}$ & $0.008_{0.0001}$ & $110.51_{0.377}$ & $2.79_{0.005}$ & $-0.0072_{0.00026}$ \\
\hline
\end{tabular}
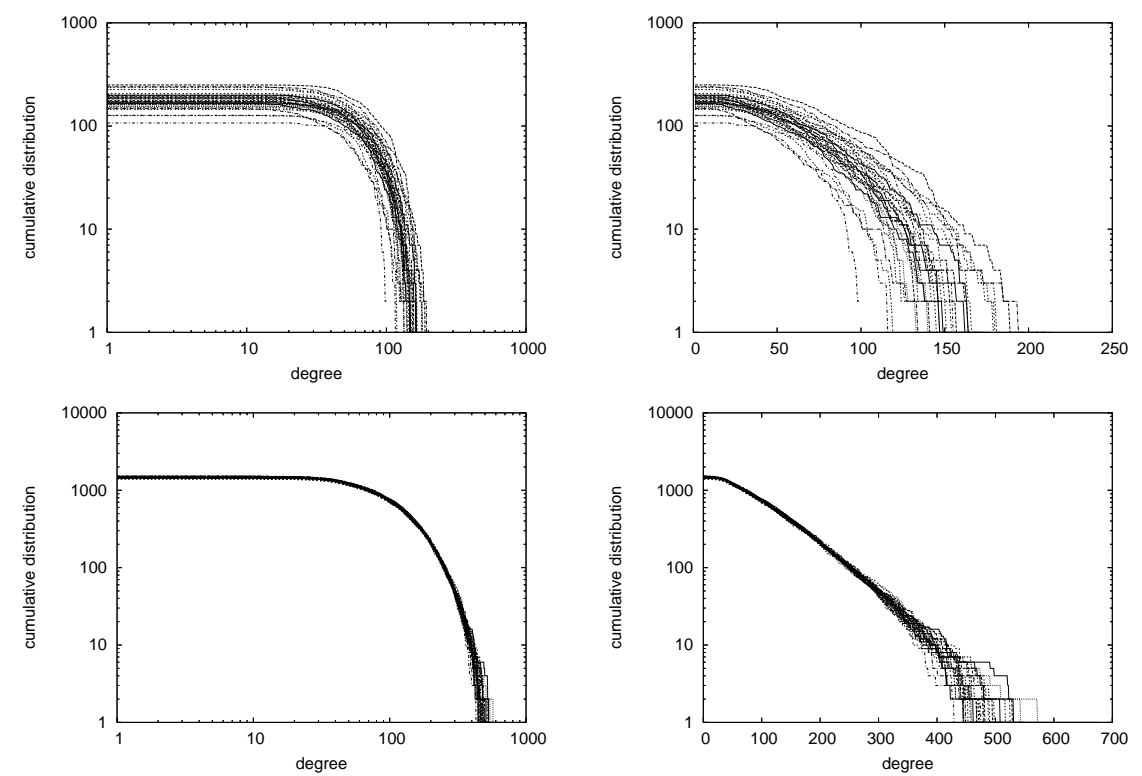

Figure 2: Cumulative degree distributions for $N=16$ and $K=4$ (top), $K=10$ (bottom). All 30 curves are plotted. Left: Log-log plot. Right: Lin-log plot.

social and technological real networks often show long tails to the right, i.e. there are nodes that have an unusually large number of neighbors. Sometimes this behavior can be described by a powerlaw, but often the distribution is less extreme and can be fitted by a stretched exponential or by an exponentially truncated powerlaw[14].

Figure 2 shows all the curves for 30 randomly generated landscapes for $N=16$ and $K=4,10$, whilst figure 3 does the same for $N=18$. To smooth out fluctuations in the high degree region, the cumulative degree distribution function is plotted, which is just the probability that the degree is greater than or equal to $k$. The sin- gle curves are shown rather than the average curve because the sum of a sufficient number of independent random variables with arbitrary distributions, provided that the first few moments exist and are finite, tends to distribute normally according to a general formulation of the central limit theorem [7]. In other words, if the average of the sum were plotted, the original shapes would essentially be lost. The curves cannot be described by power-laws: this possibility is ruled out by the left parts of figs. 2 , and 3 which are double logarithmic plots. In log-log plots, power laws should appear as straight lines, at least for a sizable part of abscissae range.

On the other hand, the right images in the same figures show that 

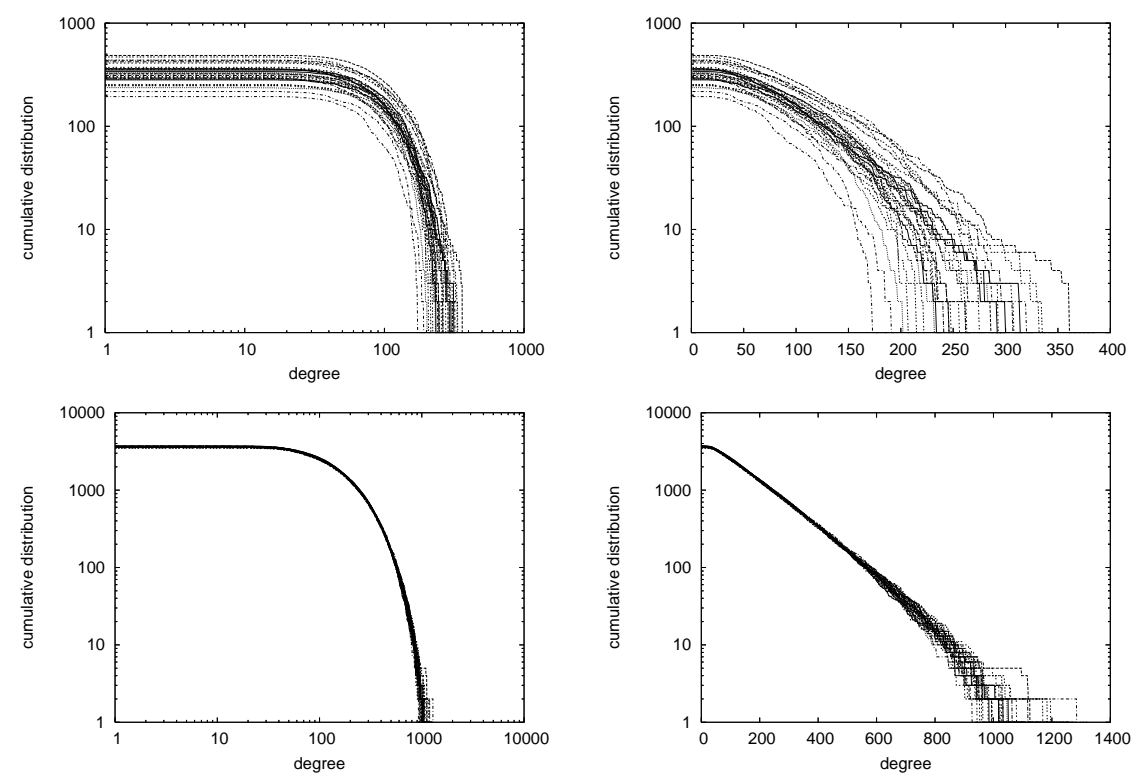

Figure 3: Cumulative degree distributions for $N=18$ and $K=4$ (top), $K=10$ (bottom). All 30 curves are plotted. Left: Log-log plot. Right: Lin-log plot.

the distributions can be fitted approximately by exponentials of the type $p(k)=(1 / z) e^{-k / z}$ where $z$ is the mean degree, as most curves are approximately straight lines on these linear-log plots. This is true for the larger part of the degree range. When we approach the finite degree cutoff the fit is obviously less good. Small networks such as those with $N=16$ and $K=4$ show larger fluctuations and their tails decay faster than exponentially. Two particular examples with a medium value of $K(K=8)$ are shown in detail in fig. A, together with an exponential fit. Table 2 gives the parameters of the regression lines for all $N$ and $K$ values.

Table 2: Correlation coefficient $(\bar{\rho})$, intercept $(\bar{\alpha})$ and slope $(\bar{\beta})$ and slope of the linear regression between the cumulative number of nodes and the degree of nodes $: \log (p(k))=\alpha+\beta k+\epsilon$. The averages and standard deviations of 30 independent landscapes, are shown.

\begin{tabular}{|c|c|c|c|}
\hline \multicolumn{4}{|c|}{$N=16$} \\
\hline$K$ & $\bar{\rho}$ & $\bar{\alpha}$ & $\beta$ \\
\hline 2 & $-0.816_{0.340}$ & $4.05_{0.717}$ & $-0.1109_{0.0379}$ \\
6 & $-0.932_{0.026}$ & $6.07_{0.276}$ & $-0.0295_{0.0026}$ \\
8 & $-0.967_{0.009}$ & $7.09_{0.105}$ & $-0.0178_{0.0009}$ \\
10 & $-0.986_{0.006}$ & $7.60_{0.107}$ & $-0.0144_{0.0007}$ \\
12 & $-0.989_{0.004}$ & $8.04_{0.125}$ & $-0.0146_{0.0008}$ \\
14 & $-0.990_{0.004}$ & $8.51_{0.156}$ & $-0.0170_{0.0010}$ \\
15 & $-0.991_{0.004}$ & $9.11_{0.121}$ & $-0.0202_{0.0010}$ \\
\hline \hline \multicolumn{3}{|c|}{$N=18$} & $-0.0220_{0.0011}$ \\
\hline 2 & $-0.823_{0.343}$ & $4.57_{0.865}$ & $-0.1088_{0.0325}$ \\
4 & $-0.951_{0.025}$ & $6.71_{0.225}$ & $-0.0198_{0.0021}$ \\
6 & $-0.982_{0.007}$ & $7.74_{0.107}$ & $-0.0098_{0.0005}$ \\
8 & $-0.991_{0.004}$ & $8.28_{0.096}$ & $-0.0076_{0.0003}$ \\
10 & $-0.994_{0.003}$ & $8.74_{0.119}$ & $-0.0076_{0.0004}$ \\
12 & $-0.995_{0.003}$ & $9.19_{0.161}$ & $-0.0088_{0.0005}$ \\
14 & $-0.995_{0.003}$ & $9.65_{0.134}$ & $-0.0110_{0.0005}$ \\
16 & $-0.994_{0.003}$ & $10.1_{0.173}$ & $-0.0139_{0.0008}$ \\
17 & $-0.994_{0.005}$ & $10.2_{0.207}$ & $-0.0151_{0.0008}$ \\
\hline
\end{tabular}
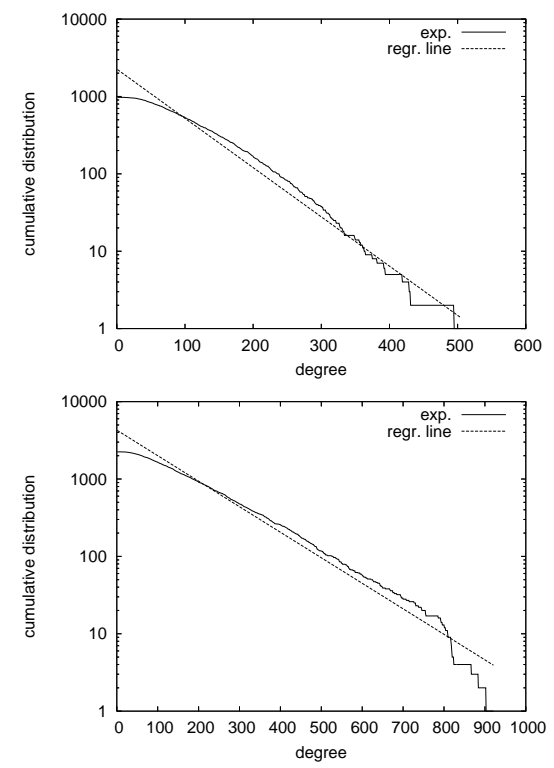

Figure 4: Cumulative degree distribution (with regression line) of two representative instances with $K=8, N=16$ (top) and $N=18$ (bottom).

If we compare these results with Doye's [5, 5] the most important difference is that we do not observe power-law distributions. Indeed, power-law degree distributions of the inherent energy landscape networks point to the "easiness" of those landscapes: due to the presence of highly connected nodes, which are also among the fittest, a simple gradient-descent would bring a searcher down to a local energy minimum, often the global one, starting anywhere in the configuration space. In other words, there exist the "funnel" 
effect described by Doye [ [ [ ] In contrast, $N K$ landscapes have tunable difficulty. How can random networks with exponential degree distributions be obtained? One way is the following: in each time step, just add a new node, and add a new link between two randomly chosen nodes, including the new one. Iterating this dynamical process produces graphs with an exponential distribution of the node degrees [ $[$ ]. But $N K$ landscapes are static and thus it is difficult to see how this process could be implemented. However, the following qualitative explanation might help. Imagine that $K$ is increased from 2 to $N-1$ in single steps. Then we could have the image of the previous landscape increasing its size and deforming itself when $K$ goes from its current value to $K+1$. The new maxima that appear could be considered as if they were added dynamically (of course some previous optima might disappear as well). Edges in the new landscape are selected essentially randomly, with more probability of selecting an already existing node. Thus, with this imaginary mechanism a distribution close to exponential would be obtained.

Thus, as the $N K$ landscape difficulty varies smoothly when $K$ is increased, the degree distribution of the corresponding maxima networks remains essentially exponential. We do not observe scalefree distributions for the easy landscapes as in the energy landscape case [f]. This is understandable: standard energy landscapes in molecular chemistry and crystal physics do correspond to thermodynamically stable states which are naturally smooth and easy to reach when the system is forming or it is slightly perturbed. In contrast, $N K$ landscape are synthetic and do not correspond to any physical principle in their construction. The only physical systems that resemble $N K$ landscapes are spin glasses, in which conflicting energy minimization requirements lead to frustration and to landscape ruggedness [11, 17]. However, disordered condensed matter systems similar to spin glasses are only obtained in particular situations, for instance by fast cooling [17].

\subsection{Basins of Attraction}

Besides the maxima network, it is useful to describe the associated basins of attraction as these play a key role in search algorithms. Furthermore, some characteristics of the basins can be related to the network features described above. The notion of the basin of attraction of a local maximum has been presented in sect. 3.1. We have exhaustively computed the size and number of all the basins of attraction for $N=16$ and $N=18$ and for all even $K$ values plus $K=N-1$. In this section, we analyze the basins of attraction from several points of view as it is described below.

\subsubsection{Global optimum basin size vs. $K$}

In Figure 5 we plot the average size of the basin corresponding to the global maximum for $N=16$ and $N=18$, and all values of $K$ studied. The trend is clear: the basin shrinks very quickly with increasing $K$. This confirms that the higher the $K$ value, the more difficult for an stochastic search algorithm to locate the basin of attraction of the global optimum

\subsubsection{Number of basins of a given size}

Figure 6 shows the cumulative distribution of the number of basins of a given size (with regression line) for two representative instances with $K=4$ and $N=16$ (top) and $N=18$. Table 3 shows the average (of 30 independent landscapes) correlation coefficients and linear regression coefficients (intercept $(\bar{\alpha})$ and slope $(\bar{\beta}))$ between the number of nodes and the basin sizes. Notice that distribution decays exponentially or faster for the lower $K$ and it is closer to exponential for the higher $K$. This observation is relevant to theoretical studies that estimate the size of attraction basins

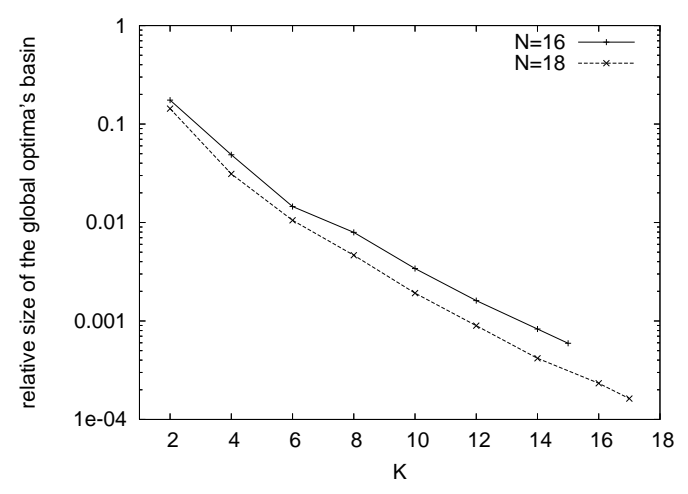

Figure 5: Average of the relative size of the basin corresponding to the global maximum for each $\mathrm{K}$ over 30 landscapes.
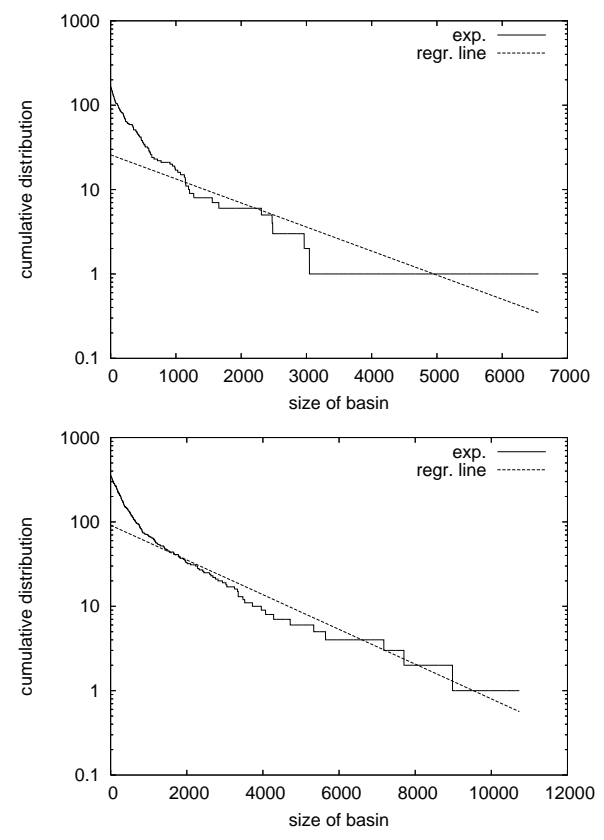

Figure 6: Cumulative distribution of the number of basins of a given size with regression line. Two Representative landscapes are visualized with $\mathrm{N}=16$ (top) and $\mathrm{N}=18$ (bottom) and $\mathrm{K}=4$. A lin-log scale is used.

(see for example [8]). These studies often assume that the basin sizes are uniformly distributed. From the slopes $\bar{\beta}$ of the regression lines (table 3 ) one can see that high values of $K$ give rise to steeper distributions (higher $\bar{\beta}$ values). This indicates that there are less basins of large size for large values of $K$. In consequence, basins are broader for low values of $K$, which is consistent with the fact that those landscapes are smoother.

\subsubsection{Fitness of local optima vs. their basin sizes}

The scatter-plots in figure日 illustrate the correlation between the basin sizes of local maxima (in logarithmic scale) and their fitness values. Two representative instances for $N=18$ and $K=4,8$ are shown. Table 1 shows the averages (of 30 independent landscapes) of the correlation coefficient, and the linear regression coefficients 
Table 3: Correlation coefficient $(\bar{\rho})$, and linear regression coefficients (intercept $(\bar{\alpha})$ and slope $(\bar{\beta})$ ) of the relationship between the basin size of optima and the cumulative number of nodes of a given (basin) size ( in logarithmic scale: $\log (p(s))=$ $\alpha+\beta s+\epsilon)$. The average and standard deviation values over 30 instances, are shown.

\begin{tabular}{|c|c|c|c|}
\hline \multicolumn{3}{|c|}{$N=16$} \\
\hline$K$ & $\bar{\rho}$ & $\bar{\alpha}$ & $\beta$ \\
\hline 2 & $-0.944_{0.0454}$ & $2.89_{0.673}$ & $-0.0003_{0.0002}$ \\
4 & $-0.959_{0.0310}$ & $4.19_{0.554}$ & $-0.0014_{0.0006}$ \\
6 & $-0.967_{0.0280}$ & $5.09_{0.504}$ & $-0.0036_{0.0010}$ \\
8 & $-0.982_{0.0116}$ & $5.97_{0.321}$ & $-0.0080_{0.0013}$ \\
10 & $-0.985_{0.0161}$ & $6.74_{0.392}$ & $-0.0163_{0.0025}$ \\
12 & $-0.990_{0.0088}$ & $7.47_{0.346}$ & $-0.0304_{0.0042}$ \\
14 & $-0.994_{0.0059}$ & $8.08_{0.241}$ & $-0.0508_{0.0048}$ \\
15 & $-0.995_{0.0044}$ & $8.37_{0.240}$ & $-0.0635_{0.0058}$ \\
\hline \hline \multicolumn{3}{|c|}{$N=18$} \\
\hline 2 & $-0.959_{0.0257}$ & $3.18_{0.696}$ & $-0.0001_{0.0001}$ \\
4 & $-0.960_{0.0409}$ & $4.57_{0.617}$ & $-0.0005_{0.0002}$ \\
6 & $-0.967_{0.0283}$ & $5.50_{0.520}$ & $-0.0015_{0.0004}$ \\
8 & $-0.977_{0.0238}$ & $6.44_{0.485}$ & $-0.0037_{0.0007}$ \\
10 & $-0.985_{0.0141}$ & $7.24_{0.372}$ & $-0.0077_{0.0011}$ \\
12 & $-0.989_{0.0129}$ & $7.98_{0.370}$ & $-0.0150_{0.0019}$ \\
14 & $-0.993_{0.0072}$ & $8.69_{0.276}$ & $-0.0272_{0.0024}$ \\
16 & $-0.995_{0.0056}$ & $9.33_{0.249}$ & $-0.0450_{0.0036}$ \\
17 & $-0.992_{0.0113}$ & $9.49_{0.386}$ & $-0.0544_{0.0058}$ \\
\hline
\end{tabular}
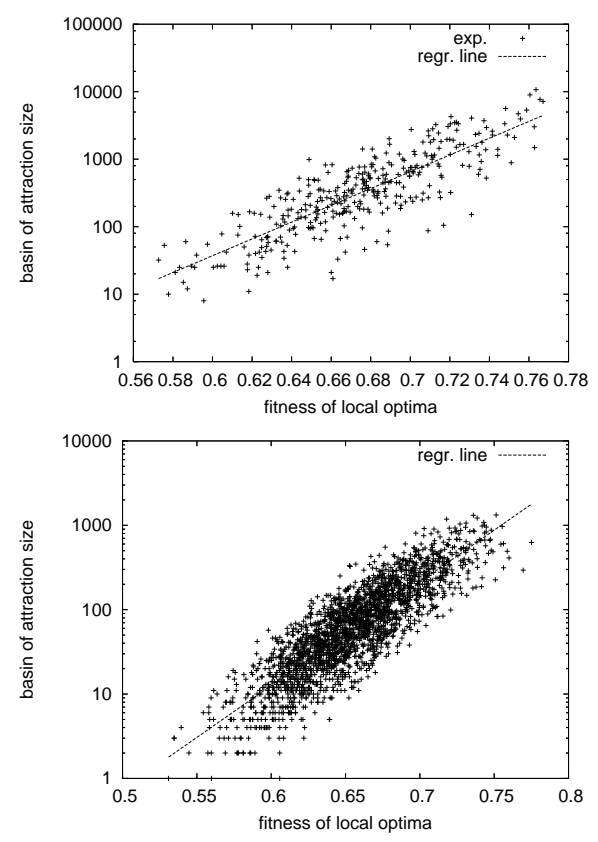

Figure 7: Correlation between the fitness of local optima and their corresponding basin sizes, for two representative instances with $N=18, K=4$ (top) and $K=8$ (bottom).

between these two metrics (maxima fitness and their basin sizes). All the studied landscapes for $N=16$ and 18, are reported. Notice that, there is a clear positive correlation between the fitness values of maxima and their basins' sizes. In other words, the higher the peak the wider tend to be its basin of attraction. Therefore, on average, with a hill-climbing algorithm, the global optimum would be easier to find than any other local optimum. This may seem
Table 4: Correlation coefficient $(\bar{\rho})$, and linear regression coefficients (intercept $(\bar{\alpha})$ and slope $(\bar{\beta})$ ) of the relationship between the fitness of optima and their basin size (in logarithmic scale: $\log (s)=\alpha+\beta f+\epsilon)$. The average and standard deviation values over 30 instances, are shown

\begin{tabular}{|c|c|c|c|}
\hline \multicolumn{3}{|c|}{$N=16^{\mid}$} \\
\hline$K$ & $\bar{\rho}$ & $\bar{\alpha}$ & $\beta$ \\
\hline 2 & $0.832_{0.0879}$ & $-15.476_{5.9401}$ & $33.066_{8.9252}$ \\
4 & $0.842_{0.0259}$ & $-13.035_{1.9907}$ & $27.094_{2.8611}$ \\
6 & $0.852_{0.0180}$ & $-12.977_{0.9921}$ & $26.061_{1.4908}$ \\
8 & $0.860_{0.0088}$ & $-12.570_{0.3769}$ & $24.880_{0.5725}$ \\
10 & $0.850_{0.0050}$ & $-11.954_{0.3501}$ & $23.561_{0.5421}$ \\
12 & $0.833_{0.0065}$ & $-11.485_{0.2993}$ & $22.519_{0.4773}$ \\
14 & $0.816_{0.0047}$ & $-11.261_{0.2008}$ & $21.864_{0.3256}$ \\
15 & $0.812_{0.0044}$ & $-11.352_{0.2109}$ & $21.876_{0.3298}$ \\
\hline \hline \multicolumn{3}{|c}{$N=18$} \\
\hline 2 & $0.839_{0.0680}$ & $-16.585_{6.0606}$ & $35.925_{8.6640}$ \\
4 & $0.842_{0.0257}$ & $-14.458_{2.1746}$ & $30.174_{3.1520}$ \\
6 & $0.852_{0.0140}$ & $-14.542_{0.9596}$ & $29.219_{1.4147}$ \\
8 & $0.867_{0.0066}$ & $-14.515_{0.3750}$ & $28.538_{0.5988}$ \\
10 & $0.866_{0.0038}$ & $-13.914_{0.3068}$ & $27.209_{0.4621}$ \\
12 & $0.854_{0.0030}$ & $-13.180_{0.1700}$ & $25.751_{0.2804}$ \\
14 & $0.836_{0.0027}$ & $-12.602_{0.1399}$ & $24.553_{0.2214}$ \\
16 & $0.822_{0.0022}$ & $-12.502_{0.1039}$ & $24.133_{0.1633}$ \\
17 & $0.817_{0.0027}$ & $-12.583_{0.1278}$ & $24.143_{0.2066}$ \\
\hline
\end{tabular}

surprising. But, we have to keep in mind that as the number of local optima increases (with increasing $K$ ), the global optimum basin is more difficult to reach by an stochastic local search algorithm (see figure 5). This observation offers a mental picture of $N K$ landscapes: we can consider the landscape as composed of a large number of mountains (each corresponding to a basin of attraction), and those mountains are wider the taller the hilltops. Moreover, the size of a mountain basin grows exponentially with its hight.

\subsubsection{Basins sizes of local optima vs. their degrees}

The scatter plots in figure 8 illustrate the correlation between basin sizes of maxima and their degrees. Representative instances with $N=18$, and $K=4,8$, are illustrated. There is a clear positive correlation between the degree and the basin sizes of maxima in the network. This observation suggests that landscapes with low $K$ values can be searched more effectively since a given configuration has many neighbors belonging to the same large basin of attraction. It is also confirmed that the basins for low $K$ are much larger than those for high $K$, not only the basin corresponding to the global maximum.

\section{CONCLUSIONS}

We have proposed a new characterization of combinatorial fitness landscapes using the well-known family of $N K$ landscapes as an example. We have used an extension of the concept of inherent networks proposed for energy surfaces [ [ $\Phi_{\text {] }}$ in order to abstract and simplify the landscape description. In our case the inherent network is the graph where the vertices are all the local maxima and edges mean basin adjacency between two maxima. We have exhaustively obtained these graphs for $N=16$ and $N=18$, and for all even values of $K$, plus $K=N-1$. The maxima graphs are small worlds since the average path lengths are short and scale logarithmically in the size of the graphs. However, the maxima graphs are not random. This is shown by their clustering coefficients, which are much larger than those of corresponding random graphs and also by their degree distribution functions, which are not 

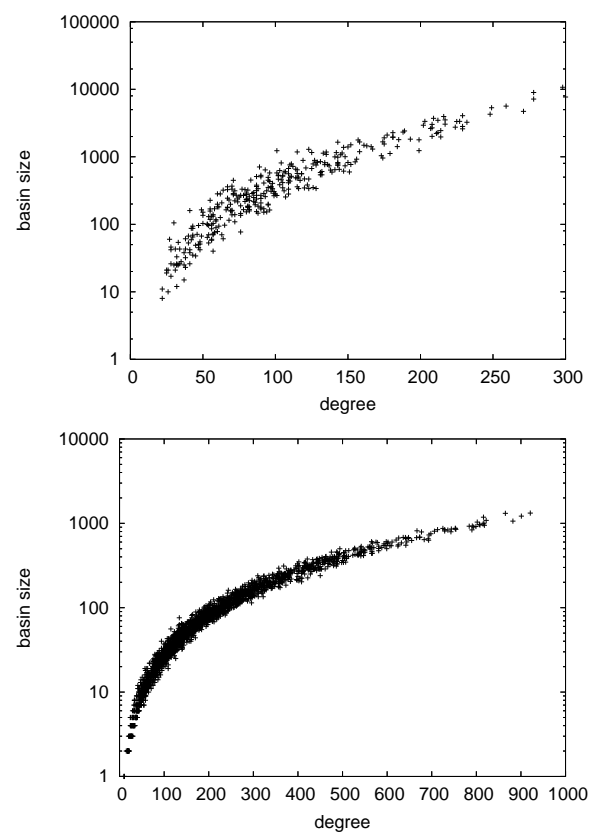

Figure 8: Correlation between the degree of local optima and their corresponding basin sizes, for two representative instances with with $N=18, K=4$ (top) and $K=8$ (bottom).

Poissonian but rather exponential. The construction of the maxima networks requires the determination of the basins of attraction of the corresponding landscapes. We have thus described the nature of the basins and their relationship with the local maxima network. We have found that the size of the basin corresponding to the global maximum becomes smaller with increasing $K$. The distribution of the basin sizes is approximately exponential for all $N$ and $K$, but the basin sizes are larger for low $K$, another indirect indication of the increasing randomness and difficulty of the landscapes when $K$ becomes large. Finally, there is a strong positive correlation between the basin size of a maxima and their degree, which confirms that the synthetic view provided by the maxima graph is a useful one.

This study represents our first attempt towards a topological and statistical characterization of easy and hard combinatorial landscapes. Much remains to be done. First of all, the results found should be confirmed for larger instances of $N K$ landscapes. This will require good sampling techniques, or theoretical studies since exhaustive sampling becomes quickly impractical. Other landscape types should also be examined, such as those containing neutrality, which are very common in real-world applications. Work is in progress for neutral versions of $N K$ landscapes. Finally, the landscape statistical characterization is only a step toward implementing good methods for searching it. We thus hope that our results will help in designing or estimating efficient search techniques and operators.

\section{ADDITIONAL AUTHORS}

\section{REFERENCES}

[1] A-L. Barabasi and R. Albert, Emergence of scaling in random networks, Science 286 (1999), 509-512.
[2] K. D. Boese, A. B. Kahng, and S. Muddu, A new adaptive multi-start technique for combinatorial global optimizations, Operations Research Letters 16 (1994), 101-113.

[3] C. Cotta and J.-J. Merelo, Where is evolutionary computation going? A temporal analysis of the EC community, Genetic Programming and Evolvable Machines 8 (2007), 239-253.

[4] S. N. Dorogovtsev and J. F. F. Mendes, Evolution of networks, Oxford University Press, Oxford, New York, 2003.

[5] J. P. K. Doye, The network topology of a potential energy landscape: a static scale-free network, Phys. Rev. Lett. 88 (2002), 238701.

[6] J. P. K. Doye and C. P. Massen, Characterizing the network topology of the energy landscapes of atomic clusters, $\mathrm{J}$. Chem. Phys. 122 (2005), 084105.

[7] W. Feller, An introduction to probability theory and its applications, Wiley, New York, 1968.

[8] J. Garnier and L. Kallel, Efficiency of local search with multiple local optima, SIAM Journal on Discrete Mathematics 15 (2001), no. 1, 122-141.

[9] M. Giacobini, M. Preuss, and M. Tomassini, Effects of scale-free and small-world topologies on binary coded self-adaptive CEA, Evolutionary Computation in Combinatorial Optimization - EvoCOP 2006 (Budapest), LNCS, vol. 3906, Springer Verlag, 2006, pp. 85-96.

[10] M. Giacobini, M. Tomassini, and A. Tettamanzi, Takeover time curves in random and small-world structured populations, Genetic and Evolutionary Computation Conference, GECCO 2005, Proceedings, ACM, 2005, pp. 1333-1340.

[11] S. A. Kauffman, The origins of order, Oxford University Press, New York, 1993.

[12] L.Luthi, M. Tomassini, M. Giacobini, and W. B. Langdon, The genetic programming collaboration network and its communities, Genetic and Evolutionary Computation Conference, GECCO 2007, Proceedings, London, England, UK (Hod Lipson et al., ed.), ACM, 2007, pp. 1643-1650.

[13] P. Merz and B. Freisleben, Memetic algorithms and the fitness landscape of the graph bi-partitioning problem, Parallel Problem Solving from Nature V (A. E. Eiben, T. Bäck, M. Schoenauer, and H.-P. Schwefel, eds.), Lecture Notes in Computer Science, vol. 1498, Springer-Verlag, 1998, pp. 765-774.

[14] M. E. J. Newman, The structure and function of complex networks, SIAM Review 45 (2003), 167-256.

[15] J. L. Payne and M. J. Epstein, Takeover times on scale-free topologies, Genetic and Evolutionary Computation Conference, GECCO 2007, Proceedings, London, England, UK (Hod Lipson et al., ed.), ACM, 2007, pp. 308-315.

[16] C. R. Reeves, Landscapes, operators and heuristic search, Annals of Operations Research 86 (1999), 473-490.

[17] D. L. Stein, Disordered sytems: mostly spin glasses, Lectures in the Sciences of Complexity (D. L. Stein, ed.), Addison-Wesley, 1989, pp. 301-353.

[18] F.H. Stillinger, A topographic view of supercooled liquids and glass formation, Science 267 (1995), 1935-1939.

[19] M. Tomassini, M. Giacobini, and C. Darabos, Evolution of small-world networks of automata for computation, Parallel Problem Solving from Nature - PPSN VIII (Birmingham, UK), LNCS, vol. 3242, Springer-Verlag, 2004, pp. 672-681.

[20] D. J. Watts, The "new" science of networks, Annual Review of Sociology 30 (2004), 243-270. 
[21] D. J. Watts and S. H. Strogatz, Collective dynamics of 'small-world' networks, Nature 393 (1998), 440-442. 